\title{
Proyecto LCweb. Respaldo documental para prácticas de laboratorio LCweb Project. Documental support for laboratory practices
}

\author{
Juan Gallego ${ }^{1}$, José Ramón Marcobal ${ }^{1}$, Ana María Rodríguez-Alloza ${ }^{2}$, Begoña Guirao ${ }^{1}$ \\ juan.gallego@upm.es, jose.marcobal@upm.es, anamaria.rodriguez.alloza@upm.es, bguirao@caminos.upm.es \\ ${ }^{1}$ Departamento de Ingeniería Civil: \\ Transporte y Territorio \\ Universidad Politécnica de Madrid \\ Madrid, España \\ ${ }^{2}$ Departamento de Ingeniería Civil: \\ Construcción, Infraestructura y Transporte \\ Universidad Politécnica de Madrid \\ Madrid, España
}

\begin{abstract}
Resumen- Las prácticas de laboratorio en enseñanza universitaria están siendo sustituidas en muchos casos por laboratorios virtuales o entornos Web que permiten trabajar a distancia. Sin embargo, parece ser que el mejor modelo docente en este ámbito está constituido por una combinación de las tradicionales prácticas presenciales en el laboratorio y laboratorios virtuales o entornos Web que complementen las prácticas reales. Esta comunicación técnica presenta el Proyecto LCweb. Se trata de un entorno Web que no sustituye sino complementa las prácticas de laboratorio de materiales de construcción de la asignatura de Firmes y Pavimentos de la Universidad Politécnica de Madrid. Su contenido está compuesto por los diferentes ensayos de laboratorio de materiales para carreteras, casos prácticos a partir de datos del laboratorio, tests de autoevaluación y normativa técnica actualizada que se utiliza en la resolución de los casos prácticos. Aunque su implantación es muy reciente, en el curso 2016/2017, todo parece indicar que los alumnos efectivamente la utilizan como complemento de las visitas al laboratorio. Proyectos de este tipo suponen un fuerte trabajo inicial, pero una vez en marcha precisan poco esfuerzo por parte del profesor y suponen un recurso importante para el alumno.
\end{abstract}

\section{Palabras clave: Prácticas de laboratorio, entorno Web, laboratorio} virtual

\begin{abstract}
Laboratory practices in the university education are being replaced for virtual laboratories or web-based applications which allow the students to work offsite. Nevertheless, it appears that the best model for this purpose is a combination of traditional hands-up laboratory work and virtual or web-based applications. This technical communication presents the LCweb Project. It is a web-based application which does not replace the hands-up work in the laboratory but complements it for the students of Pavements in the Universidad Politécnica de Madrid. The web site offers several road materials standard trials, practical examples, self-evaluation tests and the in force specifications for materials, useful to solve the practical examples. Although it has been implemented during 2016/2017 it appears that the students make use of this resource as a complement of the hands-up laboratory work rather than a substitute. This kind of projects demands a strong initial effort by the professor but when it is ongoing a small effort is required even though it gives an important support to the student.
\end{abstract}

Keywords: Laboratory practices, web-based applications, virtual laboratory

\section{INTRODUCCIÓN}

Durante las dos últimas décadas se han estudiado con detalle las ventajas e inconvenientes de sustituir los laboratorios presenciales, en los que el alumno realiza tareas y recibe indicaciones del instructor, por laboratorios virtuales y remotos.

Nedic, Machotka \& Nafalski (2003) establecían entre las ventajas del laboratorio real la interacción del alumno con el equipamiento, el trabajo colaborativo, la interacción con el instructor. Entre los inconvenientes citaba las restricciones de tiempo y espacio a las que están sujetas las prácticas presenciales en laboratorio real, las dificultades en muchas ocasiones para insertarlas en el calendario de la asignatura, sin interferir con el de otras asignaturas, el elevado coste que puede tener en instalaciones y personal, $\mathrm{y}$ en muchas ocasiones, como en el proyecto LCweb que se presenta aquí, la peligrosidad de las tareas que se realizan en el laboratorio.

En parte estas desventajas pueden verse superadas mediante la puesta a punto de laboratorios virtuales y remotos, de modo que el estudiante puede acceder a ellos sin las limitaciones anteriores.

Diversos investigadores, como Finkelstein et al. (2005) mediante estudios comparativos llegan a la conclusión de que los laboratorios virtuales no sólo superan aquellas limitaciones, sino que los alumnos que siguen prácticas de laboratorios virtuales logran una mayor adquisición de conocimientos y, lo que es más sorprendente, más habilidad en el trabajo de laboratorio.

Sin embargo, existen evidencias (Ma and Nickerson, 2006; Jara, Candelas, Puente \& Torres, 2011; Chandra \& Watters, 2001) de que los mayores logros se consiguen por métodos mixtos, es decir, que las prácticas tradicionales en laboratorio deberían combinarse con prácticas en entorno virtual o aplicaciones Web.

No obstante, muchos de los estudios realizados para determinar el efecto que estas nuevas tecnologías tienen el problema de que evalúan los avances del alumno en el laboratorio mediante la apreciación de sus habilidades (Brinson, 2015), lo que puede suponer una escala no cuantitativa, mientras que los entornos virtuales son más 
idóneos para medir cuantitativamente avances, en particular en lo que se refiere a conocimiento. Esta doble escala supone una dualidad que no hace fácil una comparación rigurosa entre las prácticas de laboratorio reales y las que se desarrollan mediante un entorno virtual.

La presente comunicación técnica se refiere al proyecto LCweb (Laboratorio de Caminos web), que se ha realizado en el Departamento de Ingeniería Civil: Transporte y Territorio de la Universidad Politécnica de Madrid, y en el que ha intervenido varios miembros de del Grupo de Innovación Educativa de Transportes.

Como se explicará en las siguientes secciones se trata de un proyecto en el que se ha optado por una vía mixta para las prácticas de laboratorio de materiales de carreteras. Por un lado se ha reducido el calendario que se dedica a las prácticas presenciales en el laboratorio universitario; por otro se ha puesto a disposición del alumno una aplicación Web que le permite disponer de documentación de respaldo para repasar lo aprendido en el laboratorio físico, mediante material multimedia, y más allá realizar una serie de casos prácticos y tests de autoevaluación. Se completa con normativa técnica actualizada. La página la puede utilizar el alumno tanto durante sus estudios como después en los primeros años de vida profesional, puesto que es de libre acceso.

Se pretende con ello optimizar la mejora del aprendizaje combinando las tradicionales prácticas presenciales de laboratorio, abreviadas, con un entorno Web que amplia y aporta un respaldo documental a la práctica presencial al laboratorio.

\section{CONTEXTO}

Hasta la entrada en vigor del nuevo Plan de Estudios de acuerdo con el R. D. 1393/2007 la asignatura de Caminos y Aeropuertos estaba situada en el quinto curso de los seis que configuraban el Plan de Estudios de Ingeniero de Caminos, Canales y Puertos. Con la entrada en vigor del nuevo Plan, los contenidos relativos a materiales para construcción de carreteras se han reunido en una nueva asignatura denominada Firmes y Pavimentos, que se imparte en la especialidad de Transportes y Servicios Urbanos. Se sitúa ahora en el cuarto curso de los cuatro que configuran el itinerario del Grado en Ingeniería Civil y Territorial. El hecho de estar localizada en el último curso la sitúa en un tramo del itinerario formativo en el que son particularmente frecuentes los trabajos, informes técnicos y prácticas que debe realizar el alumno, por lo que la inserción de las 4 prácticas de laboratorio para materiales de carreteras competía en el calendario de alumno con otras actividades igualmente recogidas en las Guías de Aprendizaje de las asignaturas que simultáneamente realiza en ese período en alumno.

Ante esta situación se optó por concentrar las cuatro prácticas que se realizaban en sólo dos prácticas, y reforzar las competencias que se logran en el laboratorio con el proyecto LCweb, una página Web dónde el alumno encuentra material audiovisual, casos prácticos, tests de autoevaluación y la normativa que precisa para, a partir de los resultados de laboratorio, tomar las decisiones que cómo facultativo le corresponden.
Es necesario aclarar que, mientras que los conocimientos teóricos que se imparten en el aula tienen como respaldo documental el libro de texto y la realización de ejercicios de oficina técnica está respaldada por un libro de problemas tipo resueltos, la formación que se recibe en el laboratorio quedaba, incluso en el Plan de Estudios anterior, sin respaldo documental.

Por tanto, con el Proyecto LCweb se han perseguido los siguientes objetivos: a) acomodar las prácticas de laboratorio a un entorno de calendario más competitivo por la concentración de actividades que tiene el alumno en ese período; b) ampliar los contenidos que permite el espacio de calendario disponible, gracias a que el alumno puede acceder en cualquier momento a este recurso; c) dotar de un apoyo documental a las prácticas de laboratorio, en formato multimedia, con posibilidad de autoevaluación, que el alumno puede utilizar durante sus estudios o después cuando inicie su vida profesional.

\section{DESCRIPCIÓN}

El entorno Web del Proyecto LCweb se ha elaborado con la herramienta Dreamweaver 13.0 de Adobe. Cuando el alumno entra a la página del Proyecto LCweb: (http://www2.caminos.upm.es/Departamentos/ict/LCweb/inici o.html) encuentra la página de inicio, con el aspecto que se observa en la Figura 1.

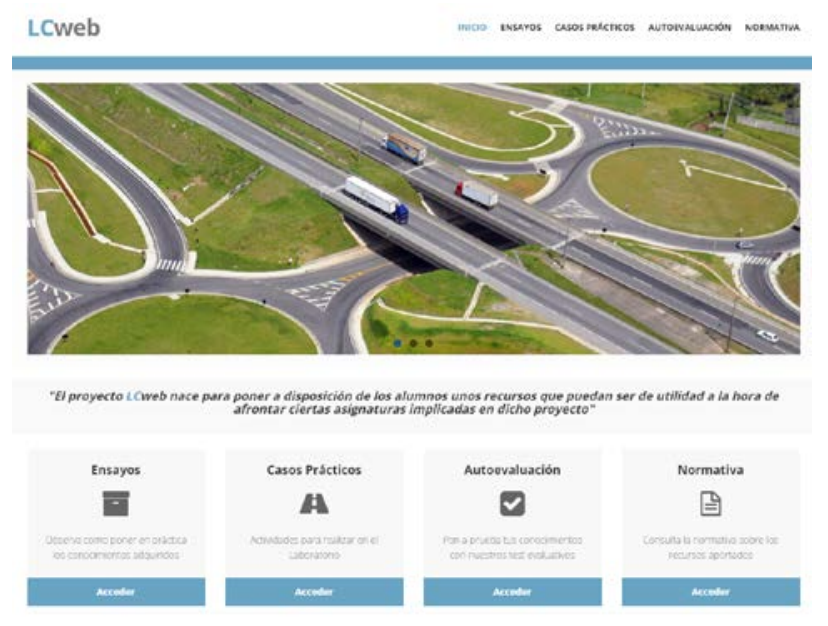

Figura 1. Página de inicio de LCweb.

Desde ella se accede a cuatro secciones: Ensayos, Casos Prácticos, Autoevaluación y Normativa.

Al abrir la sección Ensayos aparece la pantalla de la Figura 2. En ella a su vez se pueden elegir cualquiera de las cuatro prácticas que se realizaban con el Plan de Estudios antiguo y que ahora se han concentrado en sólo dos sesiones.

Si el alumno entra en la sección Áridos tendrá a su disposición un nuevo menú con los ensayos de áridos en los que ha participado en su visita física al laboratorio, e incluso otros adicionales, perfectamente explicados mediante imágenes, vídeo y texto.

Por ejemplo, si entra en el ensayo Coeficiente de los Ángeles encontrará la pantalla de la Figura 3. En este caso se cuenta con barra deslizante que permite navegar a lo largo de 
la página, viendo uno a uno los vídeos que se disponen (Figura 4). Tanto las imágenes como los vídeos están comentados por un profesor.

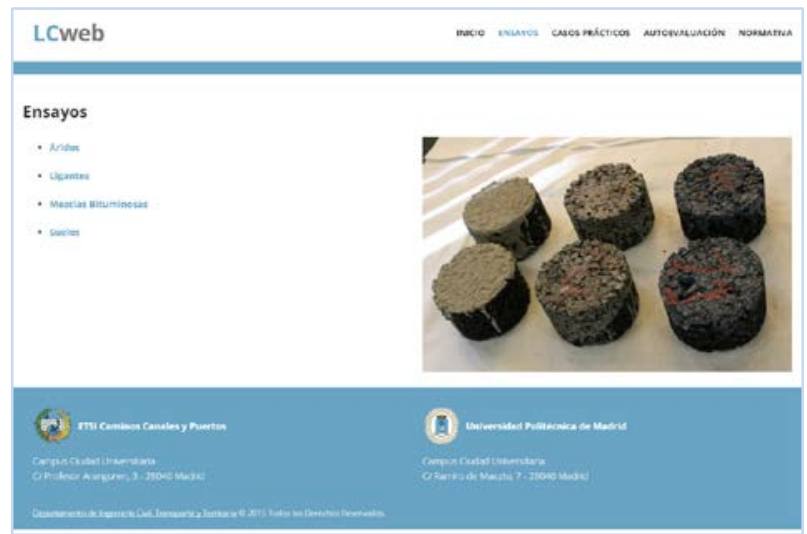

Figura 2. Menú de ensayos de materiales.

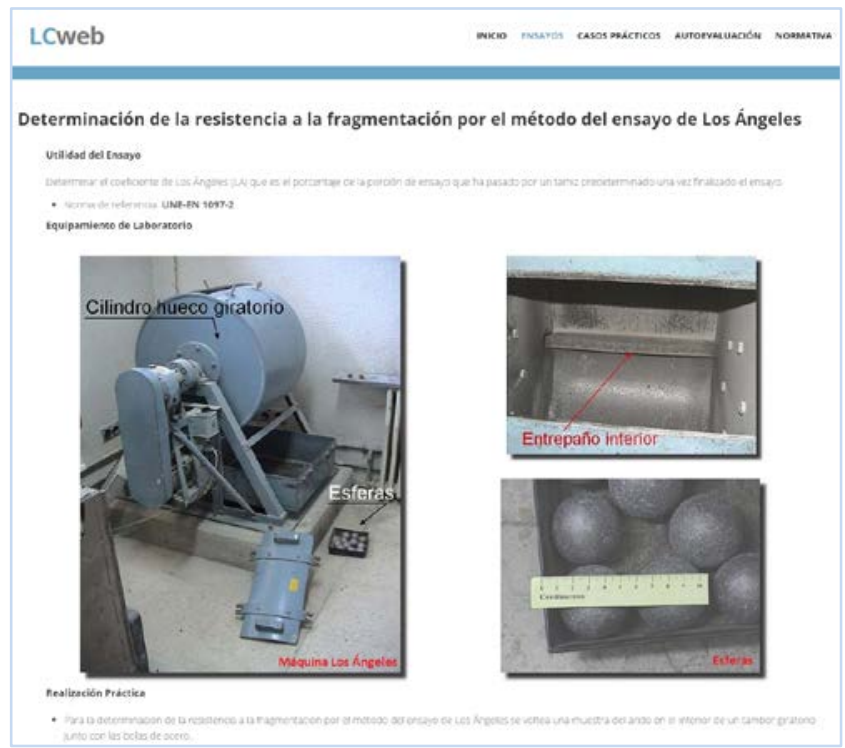

Figura 3. Fotografías comentadas. Ensayo de los Ángeles.

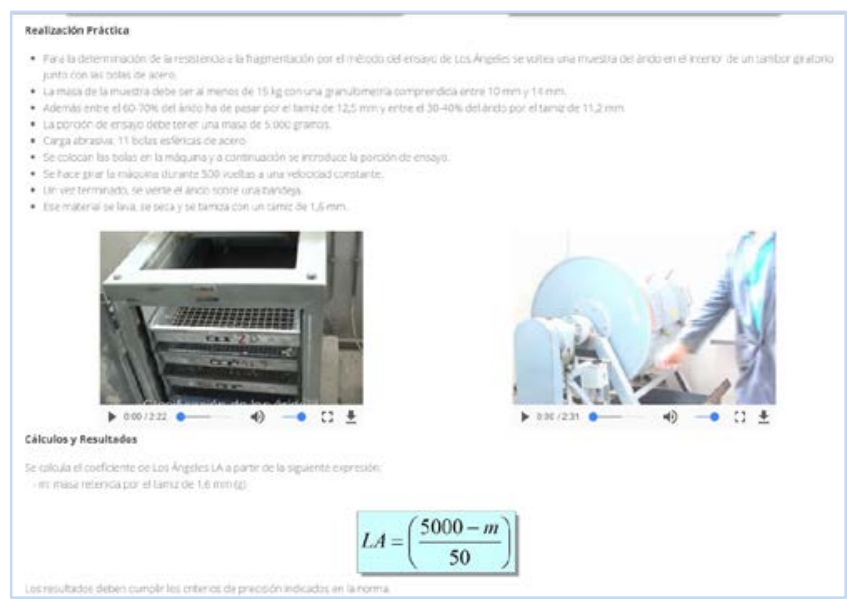

Figura 4. Videos del ensayo de los Ángeles.
Volviendo a la pantalla de inicio (Figura 1) el alumno puede acceder a la pantalla de menú de casos prácticos (Figura 5). Se trata de ficheros pdf cuyo contenido está compuesto por una serie de datos procedentes del laboratorio y refieren a un caso práctico que debe resolverse.

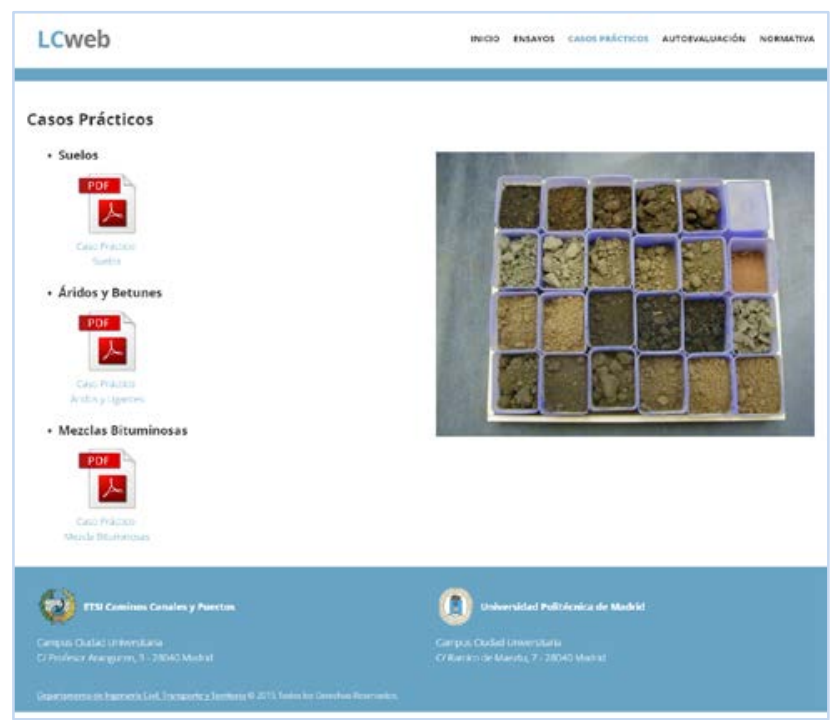

Figura 5. Menú de casos prácticos.

Desde la pantalla de inicio (Figura 1) se accede a la sección Autoevaluación (Figura 6). Allí se pueden seleccionar los distintos tests, completarlos en línea y con el botón Evaluar se obtiene la puntuación. Con el botón Obtener respuesta se muestran las soluciones correctas del test.

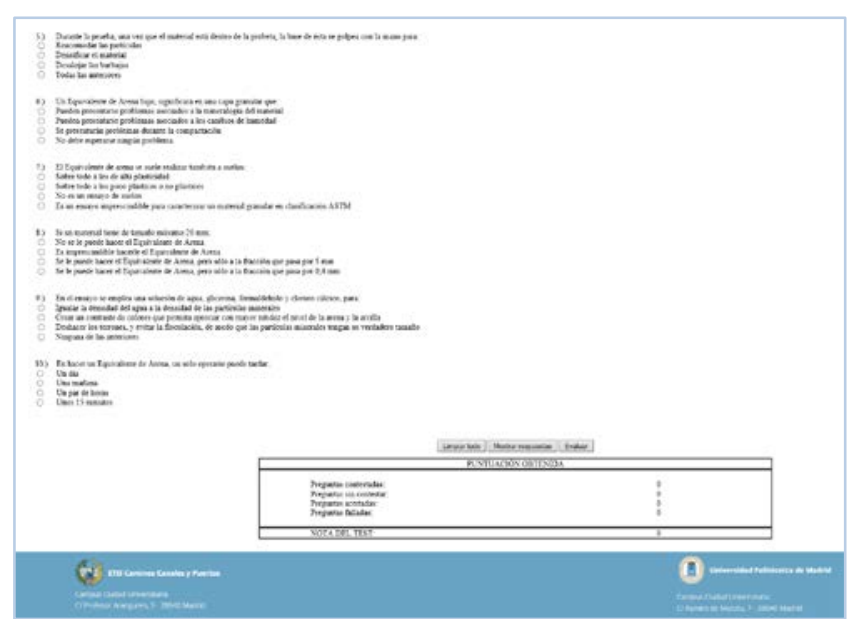

Figura 6. Test de autoevaluación.

Por último, se puede acceder a la sección Normativa, dónde el alumno encuentra la normativa actualizada de materiales para carreteras (Figura 7), que le es precisa para resolver los casos prácticos. 


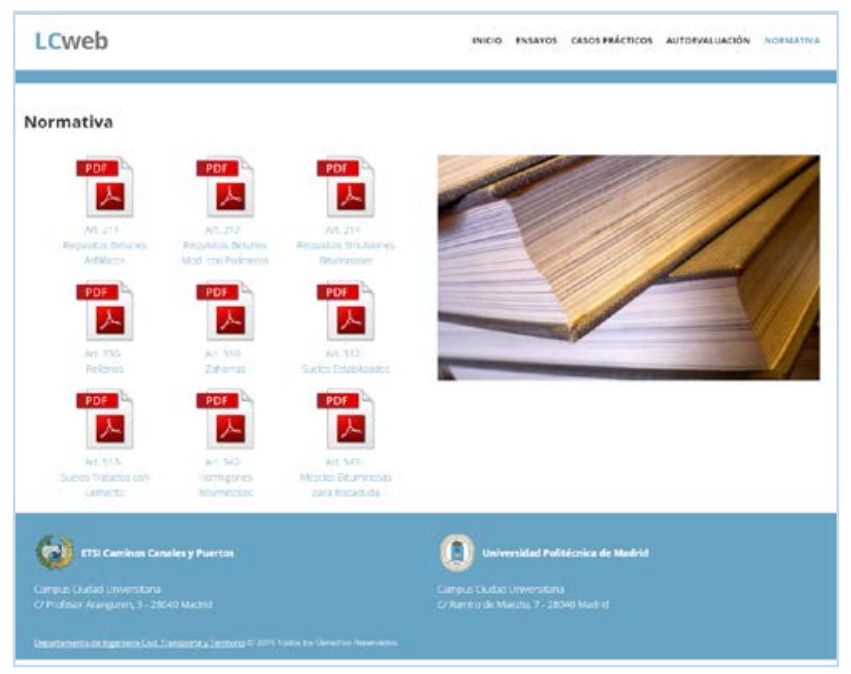

Figura 7. Menú de normativa técnica actualizada.

\section{RESULTADOS}

Actualmente se hace el seguimiento del Proyecto LCweb, aunque por lo reciente de su implantación, en el curso 2016/2017, no es posible establecer tendencias claras.

Sin embargo, sí se ha observado con claridad que los alumnos tienen el mismo nivel de participación en las prácticas presenciales de laboratorio que en cursos anteriores. Es decir, este complemento no les ha animado a ausentarse de las prácticas presenciales. Conviene aclarar que el alumno tiene como incentivo para participar en dichas prácticas un pequeño incremento en su calificación de la asignatura. La Tabla 1 presenta durante los tres últimos cursos el número de alumnos matriculados en la asignatura de Firmes y Pavimentos para la especialidad de Transportes y Servicios Urbanos, obligatoria para los alumnos de la especialidad. Se observa que el porcentaje de alumnos que ha asistido voluntariamente a las prácticas de laboratorio presenciales no ha variado sensiblemente durante el curso 2016/17, cuando se ha puesto en marcha el Proyecto LCweb, por lo que se evidencia el carácter complementario y no sustitutorio del proyecto en la formación de los alumnos.

Tabla 1. Participación en prácticas presenciales.

\begin{tabular}{|l|c|c|c|}
\hline Curso & $2014 / 15$ & $2015 / 16$ & $2016 / 17$ \\
\hline $\mathrm{N}^{\circ}$ Alumnos matriculados & 88 & 83 & 71 \\
\hline $\mathrm{N}^{\circ}$ Alumnos en prácticas presenciales & 77 & 76 & 65 \\
\hline Práctica presencial / matriculados (\%) & 89 & 92 & 92 \\
\hline
\end{tabular}

En las tutorías los alumnos frecuentemente hacen preguntas relativas a los casos prácticos que hay en LCweb, lo que evidencia que visitan la página.

Las calificaciones en la convocatoria ordinaria de Junio 2017 han sido más altas que en años anteriores, aunque puede deberse a la confluencia de varios factores, y no sólo al Proyecto LCweb. Entre el curso 2014/15 y el 2016/17 se han introducido varios cambios y mejoras como reajustes del temario que se da al alumno o la publicación de un libro de ejercicios resueltos.
Para el próximo curso está prevista una encuesta anónima a los alumnos, de modo que puedan valorar este proyecto y aportar mejoras desde el punto de vista del alumno, que beneficiarán a los alumnos de los próximos cursos.

Igualmente está previsto la inserción de un contador de accesos, distinguiendo los procedentes de la UPM, para cuantificar la utilización de la página por los alumnos, así como otros usuarios externos.

\section{CONCLUSIONES}

A partir de la bibliografía consultada sobre prácticas de laboratorio parece que el laboratorio tradicional, con presencia y participación del alumno, combinado con recursos tipo laboratorio virtual y entornos Web, constituye la mejor opción para que el alumno adquiera conocimiento y habilidades.

El Proyecto LCweb que presenta esta comunicación no ha sustituido las prácticas de laboratorio de materiales para carreteras que se venían impartiendo hasta ahora. Ha permitido reducir la duración de aquéllas puesto que la nueva herramienta amplía el número de actividades que el alumno realiza en el laboratorio, le da acceso a casos prácticos y le permite su propia autoevaluación. Se trata de una documentación multimedia que respalda documentalmente las actividades del laboratorio y las amplía.

El contenido de LCweb incluye la explicación detallada de ensayos de suelos, áridos, ligantes y mezclas bituminosas; casos de aplicación práctica a partir de resultados de laboratorio; tests de autoevaluación y normativa actualizada.

Este tipo de herramientas puede aplicarse en general en las enseñanzas del área de construcción, aunque es necesario advertir que el trabajo inicial para poner en marcha el recurso es muy costoso en cuanto a horas de dedicación. No obstante, una vez elaborado, su sostenibilidad requiere poca dedicación: actualización de los ensayos o de la normativa cuando se produzcan cambios en el sector.

Conviene por último señalar que en los casos en los que por motivos presupuestarios una institución universitaria no disponga de laboratorio adecuado, un proyecto de este tipo puede contribuir no ya a completar la formación presencial en el laboratorio sino incluso a sustituirla, aunque sería de manera deficiente desde nuestra experiencia de años.

\section{AGRADECIMIENTOS}

Esta comunicación técnica se ha desarrollado dentro de las actividades de investigación del Grupo de Innovación Educativa en Transportes de la UPM (GIE TRANSPORTS). En concreto, esta investigación se enmarca dentro del Proyecto de Innovación educativa IE1617.0401, financiado por la UPM en la convocatoria competitiva de 2016-2017.

\section{REFERENCIAS}

Brinson, J.R. (2015) Learning outcome achievement in nontraditional (virtual and remote) versus traditional (handson) laboratories: A review of the empirical research. Computers and Education 87, 218-237. Doi:10.1016/j.compedu.2015.07.003 
Chandra V. \& Watters J.J. (2011). Re-thinking physics teaching with web-based learning. Computers \& $\begin{array}{lll}\text { Education } & 58 & \text { (2012) }\end{array}$ doi:10.1016/j.compedu.2011.09.010

Finkelstein, N. D., Adams, W. K., Keller, C. J., Kohl P. B., Perkins. K.K., Podolefsky, N. S., \& Reid, S. (2003). When learning about the real world is better done virtually: A study of substituting computer simulations for laboratory equipment. Physics Education Research 1, 010103. doi:10.1103/PhysRevSTPER.1.010103

Jara C.A., Candelas, F.A., Puente S.T. \& Torres F. (2011). Hands-on experiences of undergraduate students in
Automatics and Robotics using a virtual and remote laboratory. Computer s \& Education 57 (2451-2461) doi:10.1016/j.compedu.2011.07.003

Ma, J, \& Nicherson, J.V. (2006). Hands-On, Simulated, and Remote Laboratories: A Comparative Literature Review. ACM Computing Surveys. Vol. 38, No. 3, Article 7. doi:10.1145/ 1132960.1132961

Nedic, Z., Machotka, J. \& Nafalski, A. (2003). $33^{\text {rd }}$ ASEE/IEEE Frontiers in Education Conference. Boulder, CO 